\title{
The long term experience of thymectomy for myasthenia gravis
}

\author{
GK SCADDING, CWH HAVARD, MJ LANGE, I DOMB
}

From the Departments of Endocrinology and Neurological Science, Royal Free Hospital, London.

SUMMARY A retrospective survey of the records of 287 patients with generalised myasthenia gravis treated at New End Hospital and later at the Royal Free Hospital by anticholinesterase drugs, with or without thymectomy, between the years 1942 and 1976 , shows that $62 \%$ of patients were improved. The timing of the operation, the grading of disease and the age and sex of the patient did not greatly influence overall results. The poor prognosis of thymic tumours was confirmed in this series. A decrease occurred in the number of patients achieving complete remission after 1961, although the proportion of patients improving did not fall. It is possible that anticholinesterase therapy may alter the response to thymectomy.

Myasthenia gravis is an auto-immune disease in which muscle weakness occurs because the number of functioning acetylcholine receptors (AChRs) is reduced by auto-antibodies (anti-AChRs). ${ }^{1}$ Thymectomy was first performed on a myasthenic patient in $1911,{ }^{2}$ with some improvement in her condition. In 1936 Blalock $^{3}$ removed a thymic cyst from a girl whose myasthenia subsequently improved dramatically. A further six patients were operated upon and within a month three no longer required anticholinesterases. The first thymectomy in England was performed by Sir Geoffrey Keynes in 1942, at New End Hospital, now part of the Royal Free Hospital. He published his results in 1946,1954 and $1955,,^{4-7}$ noting that thymectomy was of greater benefit in patients without thymomas. The operation is now widely accepted as an important part of the treatment of myasthenia gravis with benefit to some $60 \%$ of patients. ${ }^{8}$

In recent years the therapeutic use of corticosteroids and azathioprine has made the effects of thymectomy alone more difficult to evaluate. Controversy still exists about the importance of such features as age, sex, duration of disease and thymic histology in determining the magnitude and rapidity of the response to treatment which may take several years to become manifest. As long ago as 1960 Lange suggested that the sooner thymectomy is per-

Address for reprint requests: Dr GK Scadding, The Middlesex Hospital Medical School, Tottenham St, London WC.

Received 11 November 1983 and in revised form 10 October 1984. Accepted 12 October 1984 formed following the onset of the disease the better the prognosis. ${ }^{8}$ Olanow et $a l^{\varphi}$ have suggested recently that the response to thymectomy is more rapid and complete if the operation is performed early in the course of the disease and if the patient does not receive cholinesterase inhibitors. These drugs (pyridostigmine, neostigmine) are the mainstay of symptomatic treatment for myasthenia and act by inhibiting the enzyme cholinesterase which normally hydrolyses acetyl choline (ACh) at the neuromuscular junction. The quanta of $\mathrm{ACh}$ released by the nerve terminal are therefore able to interact with the available AChRs for longer and the chances of a resulting muscle action potential are increased. The dosage is critical: weakness ensues if excess anticholinesterase is used. Furthermore chronic administration of anti-cholinesterase to normal animals results in changes at the neuromuscular junction similar to those seen in myasthenia and comprising simplification of the motor end plate with loss of folds. ${ }^{10}$

The rationale behind thymectomy remains unknown. Thymic lymphocytes from over $70 \%$ of patients with myasthenia gravis and thymitis spontaneously synthesise anti-AChR in vitro, " suggesting that they are already primed and that in vivo antibody production takes place in the thymus. Moreover, thymic cells enhance anti- $\mathrm{AChR}$ production by autologous peripheral blood lymphocytes, suggesting that the thymus contains a factor or factors such as $\mathrm{AChR}$ bound to antigen presenting cells, which stimulate the formation of anti-AChRs in vivo. ${ }^{12} \mathrm{AChR}$ has been demonstrated in the 
human myasthenic thymus using peroxidaseconjugated alpha-bungarotoxin, ${ }^{13}$ a snake venom which binds specifically and irreversibly to AChRs.

It is conceivable that anticholinesterase-induced damage to the neuromuscular junction could result in the release of AChRs in an antigenic form and that this could perpetuate the state of autoimmunity, even after removal of a thymic source of AChR antigen.

Since we have at our hospital the records of a large number of patients treated by thymectomy, without corticosteroids or azathioprine, who received varying doses of anticholinesterases, it appeared worthwhile to analyse these results. It must be acknowledged that they are retrospective, not cross-matched and based in part on subjective assessment.

\section{Patients and methods}

Three hundred and ninety eight patients with myasthenia gravis were seen at New End Hospital and later at the Royal Free Hospital, between 1942 and 1976. Of these 21 were lost to follow up, of the remainder four had congenital myasthenia, the diagnosis was uncertain in 39 , a further 14 underwent thymectomy elsewhere and 33 received immunosuppression after thymectomy, leaving 287 patients for assessment. The records of these 287 patients have been examined. Only patients with undoubted myasthenia gravis were included and only those patients who had not received corticosteroids or azathioprine. All these patients have been either examined or corresponded with at yearly intervals and their status in $\mathbf{1 9 8 0}$ was assessed as follows:

Category 1 Able to do a full days work without muscle weakness. No anti-cholinesterase medication needed. Also included were patients who had fulfilled these criteria for more than 5 years but had later relapsed.

Category 2 Improved, either in that work capacity increased, or their medication decreased, or both. Anticholinesterases still needed.

Category 3 Unchanged.

Cateogry 4 Worse or dead. (Where death was clearly unassociated with myasthenia gravis, for example car crash, the category to which their myasthenia gravis belonged was used.)

Patients first seen after 1976 were excluded because this allowed insufficient time to respond to thymectomy. The $\chi^{2}$ test of significance was employed to evalute results.
Table 1 State of 287 patients at time of review

\begin{tabular}{lll}
\hline Patient state & $\begin{array}{l}\text { Thymectomy } \\
(n=243)\end{array}$ & $\begin{array}{l}\text { No thymectomy } \\
(n=44)\end{array}$ \\
\hline Category 1 & $25.9 \%$ & $11.4 \%$ \\
Category 2 & $42.3 \%$ & $16.0 \%$ \\
Category 3 & $5.0 \%$ & $29.5 \%$ \\
Category 4 & $26.7 \%$ & $43.2 \%$ \\
\hline
\end{tabular}

\section{Results}

Two hundred and eighty seven patients were available for assessment, of whom 243 had undergone thymectomy. Of the operated group $68.2 \%$ were in categories 1 and 2, 5\% were unchanged and $26.7 \%$ worse or dead. In the non-operated group, these figures were $27.4 \%, 29.5 \%$ and $43.2 \%$ (table 1 ).

The results of operation are analysed in 5 year blocks (table 2), and it can be seen that although the proportion of patients achieving category 1 remains fairly constant until 1961 , it then drops to a lower level which is maintained. The proportion of patients improving (that is achieving categories 1 and 2) does not show this change. Analysis of the figures on a $\chi^{2}$ table gives a highly significant value of $p<0.001$.

\section{State 5 years after operation}

Other factors may influence the response to operation. It is conceivable that amongst those operated on recently, the number of patients in Category 18 could still increase, since there is a tendency for the number of patients entering Category 1 to increase with time (fig) some patients taking as long as twenty years. In order to avoid this bias the number of patients in Category 1 five years after operation was assessed (table 4). Again there is a higher proportion in the earlier years, with a decrease occurring by the 1952-1956 period, though this is less sharply defined.

\section{Duration of disease}

The duration of disease before thymectomy may be of importance. Between 1942 and 1946, and 1947 and 1951, there appears to be an effect with more patients entering remission if operation is performed early in the course of the disease, $\chi^{2}=11.83$ and $9.54, p<0.05$ for both. How-

Table 2 Results of thymectomy analysed in 5 year blocks

\begin{tabular}{lcccc}
\hline Year of operation & \% in category 1 & \% improving (Categories 1 \& 2) & \% thymomas & $\begin{array}{c}\text { No of patients } \\
\text { not operated }\end{array}$ \\
\hline $1942-46(\mathrm{n}=52)$ & $30 \cdot 8$ & $51 \cdot 9$ & $15 \cdot 4$ & 7 \\
$1947-51(\mathrm{n}=68)$ & $35 \cdot 3$ & $76 \cdot 5$ & $8 \cdot 8$ & 5 \\
$1952-56(\mathrm{n}=42)$ & $28 \cdot 6$ & $61 \cdot 9$ & $19 \cdot 0$ & 12 \\
$1957-61(\mathrm{n}=18)$ & $27 \cdot 8$ & $88 \cdot 9$ & $25 \cdot 0$ & 3 \\
$1962-66(\mathrm{n}=24)$ & $8 \cdot 3$ & $54 \cdot 2$ & $29 \cdot 0$ & 6 \\
$1967-71(\mathrm{n}=22)$ & $9 \cdot 0$ & $77 \cdot 3$ & $17 \cdot 6$ & 6 \\
$1972-76(\mathrm{n}=17)$ & $11 \cdot 8$ & $88 \cdot 2$ & & \\
\hline
\end{tabular}




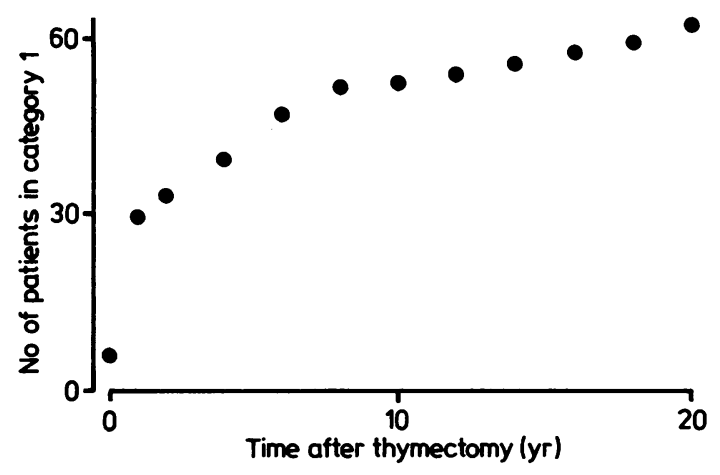

Fig Number of patients in Category 1 plotted against years since thymectomy.

ever in subsequent years there is no such correlation, nor is there any correlation between the time to thymectomy and the time to remission in those patients entering Category 1.

The sex of the patient has no effect in this series, $\chi^{2}=$ $0.52 \mathrm{p}$ (NS), neither does the grading of the myasthenia (Osserman classification) ${ }^{14}$ prior to operation.

\section{Histology (table 5)}

Unfortunately, the effect of thymic histology upon the response to thymectomy is impossible to evaluate in detail since the sections are no longer available for analysis by a single individual and reports from earlier years are lacking in detail. However, if those patients with thymomas are considered, their prognosis is obviously worse, $\chi^{2}=29$, $\mathrm{p}<0.001$, although $10 \%$ did achieve Category 1 , for at least 5 years after operation. Two of these relapsed and died suddenly; in one there was no evidence of thymoma or tumour at necropsy. The percentage of patients with thymomas in each 5 year period is shown in table 2 . The majority of thymomas were lympho-epitheliomas; however, one patient had a benign neurilemmoma in his thymus and improved after its removal.

\section{Anti-cholinesterase therapy}

Pyridostigmine (Mestinon) was first used at the Royal Free Hospital in 1955 and over the next few years most patients were changed to this drug from neostigmine, because of its longer duration of action. Although it is difficult to obtain a detailed drug history from records, the patients have been divided into two categories. Those who received neostigmine only before operation and those who received pyridostigmine with or without neostigmine. The proportion in each category is shown in table 3a. Again the proportion of patients improving is rather similar $(71.7 \%$ and $67 \%$ ) but in those patients who received pyridostigmine there are fewer in Category $1, x^{2}=6.9, \mathrm{p}<0.05$.

When the dosage of anticholinesterase received was considered by dividing the patients into those taking less than or equal to $360 \mathrm{mg}$ pyridostigmine (or the equivalent $90 \mathrm{mg}$ neostigmine) daily, and those exceeding this dose the proportions attaining each category are shown in table $3 \mathrm{~b}$. The percentage of patients improving is similar $(65.2 \%$ and $69.6 \%$ ). There is a suggestion that the lower drug dosage is associated with an increased proportion $(32.6 \%$ as opposed to $21.4 \%$ ) of patients achieving Category 1 , but this fails to reach statistical significance $\chi^{2}=4 \cdot 32, p<0 \cdot 2$.

\section{Late relapse}

Late relapses were noted in a few individuals, notably the first patient to undergo thymectomy in England. She went into remission 4 years after operation and remained well, working as a land girl for 17 years. However relapse occurred when she had pneumonia in 1963 and for the remaining 16 years of her life she required anticholinesterase. Of eight patients with periods of 7-36 years (mean 15.8 \pm 8.5 ) spent in remission, relapse was precipitated in three by chest infections and in one by emotional stress. Three have subsequently gone back into remission. Of these eight patients, seven had undergone thymectomy, the eighth had a long spontaneous remission which was not repeated. On enquiring into the history, a few patients suffered an episode of transient weakness, usually ocular, occurring several years before the "onset" of myasthenia gravis, suggesting that the disease can remain latent for a long period.

\section{Radiotherapy}

Of the thymectomy group 19 patients received radiotherapy to the thymus, in all but one of these a thymoma was present. The distribution of these patients between the various categories follows closely that of the patients with thymomas as a whole. Five of the nonoperated group received radiotherapy and one was in Category 1 , one in 2 and three were dead at assessment.

Table 3a Results of patients grouped according to type of medication received

\begin{tabular}{llll}
\hline Treatment & $\%$ in category 1 & Category 2 & Category 3 and 4 \\
\hline $\begin{array}{l}\text { Pyridostigmine } \pm \text { neostigmine }(\mathrm{n}=69) \\
\text { Neostigmine }(\mathrm{n}=146)\end{array}$ & $15 \cdot 4$ & $56 \cdot 3$ & $28 \cdot 2$ \\
\hline
\end{tabular}

Table 3b Results of patients analysed according to dose of anticholinesterase received

\begin{tabular}{|c|c|c|c|}
\hline Dose of anticholinesterase & $\%$ in category 1 & Category 2 & Category 3 and 4 \\
\hline $\begin{array}{l}\text { Less than or equal to } 360 \mathrm{mg} \text { pyridostigmine } \\
\text { ( } 90 \mathrm{mg} \text { prostigmine) daily }(\mathrm{n}=49) \\
\text { More than the above dose }(\mathrm{n}=168)\end{array}$ & $\begin{array}{l}32.6 \\
21.4\end{array}$ & $\begin{array}{l}32.6 \\
48.2\end{array}$ & $\begin{array}{l}34 \cdot 7 \\
30.4\end{array}$ \\
\hline
\end{tabular}


Table 4 Percentage of patients achieving category 1 within 5 years of operation

\begin{tabular}{ll}
\hline & $\%$ \\
\hline $1942-46$ & 23 \\
$1947-51$ & 25 \\
$1952-56$ & $14 \cdot 3$ \\
$1957-61$ & 11 \\
$1962-66$ & $8 \cdot 3$ \\
$1967-71$ & $4 \cdot 5$ \\
$1972-76$ & $5 \cdot 9$ \\
\hline
\end{tabular}

\section{Deaths}

The per-operative death rate (defined as death occurring within 1 month of operation) fell from $15.4 \%$ of all patients operated on between 1942 and 1946 to less than $5 \%$ after 1956 , with no per-operative deaths in the periods 1957-1961 and 1972-1976 (table 6). The total number of deaths in the earlier years is biased by the long time interval since deaths have been assumed to be myasthenia gravis related unless there is evidence to the contrary. Therefore, the number of patients dead 5 years after operation is considered. Patients with thymomas account for between almost half and three quarters of all myasthenia gravis associated deaths in each five year group demonstrating again the poor prognosis that a tumour conveys.

In the non-operated group, 17 of the 44 patients $(38.6 \%)$ were dead with a mean survival time from onset of disease of $5 \cdot 2 \pm 5$ years. Of the eight patients with probable thymomas (based on radiological evidence and positive striated muscle antibodies) who were not operated upon, seven are dead. Their mean survival time from onset of symptoms was $5 \cdot 3 \pm 5 \cdot 4$ years.

\section{Discussion}

The controversies concerning myasthenia gravis and its treatment have been reviewed recently. ${ }^{15}$ Thymectomy is generally accepted as being effective in $66-86 \%$ of patients in whom there is no thymoma, although the rationale for the operation is still not known. The possibility of deleterious effects from anticholinesterases has been raised. ${ }^{15}$ The doses of neostigmine used in animal experiments that have demonstrated damage to the neuromuscular junction were much larger than the doses administered to patients and there is no clinical evidence of declining effect of anticholinesterase drugs over the years. This has however never been formally assessed.

Any retrospective assessment of the results of thymectomy is bedevilled by several factors: the operated and non-operated patients are not matched for age, sex, disease severity and duration; the improvement in surgery, anaesthesia and postoperative care may alter results in recent years; patient assessment is difficult and some may be lost to follow up; the modes of treatment may have altered. Our study has attempted to take these into account as far as possible. No comparison is feasible between operated and non-operated patients because the two populations are different: those not operated upon were either too old, had associated disease, very mild myasthenia gravis or refused operation. The possibility that thymectomy is ineffective and that the remissions following operation are entirely spontaneous has been raised, ${ }^{16}$ but 0 appears unlikely in view of the low rate $(16 \%)^{1718}$ and short duration (average 2.2 years) ${ }^{19}$ of spon- $ڤ$ taneous remissions reported in myasthenia gravis $\underset{\perp}{\stackrel{1}{2}}$ before thymectomy was widely practised. The treatment policy at the Royal Free varied little between 1945 and 1976, with most patients being offered thymectomy soon after they were seen unless their disease was very mild or they were unfit for a general anaesthetic because of extreme age or other disease. No patients were given immunosuppressive therapy as an alternative to surgery. An improvement in per-operative mortality has occurred, thus more of the severely affected patients

Table 5 Results of patients analysed according to thymic histology

\begin{tabular}{lcll}
\hline Thymic histology & \% in category 1 & Category 2 & Category 3 and 4 \\
\hline Thymoma $(\mathrm{n}=42)$ & $9 \cdot 5$ & $23 \cdot 8$ & $66 \cdot 7$ \\
Non thymoma $(\mathrm{n}=201)$ & 29.4 & 46.3 & $24 \cdot 3$ \\
\hline
\end{tabular}

Table 6 Death rates after thymectomy

\begin{tabular}{llll}
\hline Date of thymectomy & $\begin{array}{l}\text { Per-operative deaths as \% } \\
\text { of total no. operated on }\end{array}$ & $\begin{array}{l}\text { \% with myasthenia gravis } \\
\text { associated death within } 5 \text { years } \\
\text { of thymectomy }\end{array}$ & $\begin{array}{c}\text { \% of patients dead within } 5 \text { years } \\
\text { who had a thymoma }\end{array}$ \\
\hline $1942-46$ & $15 \cdot 4$ & $30 \cdot 8$ & $43 \cdot 8$ \\
$1947-51$ & $7 \cdot 4$ & $16 \cdot 0$ & $54 \cdot 5$ \\
$1952-56$ & $16 \cdot 7$ & $28 \cdot 6$ & $50 \cdot 0$ \\
$1957-61$ & 0 & $5 \cdot 5$ & $100 \cdot 0$ \\
$1962-66$ & $4 \cdot 2$ & $29 \cdot 2$ & $57 \cdot 0$ \\
$1967-71$ & $4 \cdot 5$ & $13 \cdot 6$ & $100 \cdot 0$ \\
$1972-76$ & 0 & 0 & 0 \\
\hline
\end{tabular}


probably survived operation in recent years: however, there is no correlation between severity of disease at operation and state at 5 years. The surgeons undertaking thymectomy have changed but the same operative techniques have been continued, total removal of all possible thymic tissue being attempted through a sternal split and all operators have been highly skilled and competent. A change in surgical technique, or failure to remove the whole thymus might be expected to result in a decrease in the proportion of patients improving and this is not seen. Patients have been divided into very broad categories and the majority have been clinically assessed by one of us (MJL). Only a small proportion (5\%) have been lost to follow up.

Category 1 in this paper cannot equate with complete remission since some of the assessments were subjective. However recent evidence suggests that some patients in objective clinical remission still have evidence of myasthenia gravis; in our initial report three of four such patients had raised antiAChR titres ${ }^{20}$ and Emeryk et al have shown that only five of 16 patients had normal single fibre electromyography findings. ${ }^{21}$

Our results agree broadly with those of Simpson. ${ }^{18}$ As in his study both sexes derive benefit from the operation and are equally distributed among the categories afterwards; patients with thymomas have a poorer prognosis; (one in three survived in Simpson's series; two out of three were in Categories 3 and 4 in ours), but those who improve can achieve category 1. Improvement was most evident in Simpson's series when the duration of the disease at the time of operation was less than 5 years; this effect is only evident in the 1942-46 and 1947-51 periods in our study, when neostigmine was used. Patients who had their operation at a younger age than average showed maximum improvement in Simpson's study; in ours more patients operated on under the age of 20 appear in Category 1 than would be expected, (40\% as opposed to $30 \%$ ) but the effect of age at operation does not reach statistical significance, $\chi^{2}=$ $22, p>0 \cdot 1$. Simpson used the pre-operative dose of neostigmine as an indication of disease severity and showed that death from myasthenia was more probable in those patients requiring large doses, but if they survived the post-operative category was not affected by disease severity. In our patients also pre-operative findings as judged by Osserman classification ${ }^{14}$ did not influence the outcome.

Our finding that the proportion of patients completely recovering after thymectomy decreased from about the 1960 s, despite the fact that there was no concomitant fall in the proportion of patients improving could be significant, especially when considered in relation to the gradual change of anti- cholinesterase therapy from 1955 onwards. Patients who received neostigmine only appeared to have a better chance of entering Category 1 than those who received pyridostigmine or a combination of the two. Although it is conceivable that anticholinesterases, which are known to have deleterious effects at the neuromuscular junction, could alter the response of myasthenia gravis patients to thymectomy, it is difficult to envisage how this could be true for one form of the drug and not another. In our patients this effect was more strongly correlated with the nature of the anticholinesterase than with the dosage. However the relationship between oral intake of cholinesterase and blood levels varied greatly between individuals. ${ }^{22}$ Roses (personal communication) reports that 20 patients treated by thymectomy without any prior anti-cholinesterase medication are now all normal.

Other factors could be responsible for the fall in Category 1 patients: the incidence of thymomas varies in each five year block, but it is not of sufficient magnitude to account for the decreased numbers in Category 1 . The association of a better prognosis with neostigmine treatment may be because of another unidentified factor operating in the earlier years of thymectomy, when neostigmine was the only anticholinesterase available.

We have already drawn attention to the possible adverse effects of azathioprine in myasthenia gravis. ${ }^{23}$ We should like to suggest that long term treatment with pyridostigmine could also be harmful and that other centres should examine their records to corroborate or refute this observation.

\section{References}

' Lindstrom JM, Seybold ME, Lennon VA, Whittingham S, Duane D. Antibody to acetylcholine receptor in myasthenia gravis. Prevalence, clinical correlates and diagnostic value. Neurology (Minneap) 1976;26: 1054-9.

${ }^{2}$ Schumacher M, Roth J, Thymectomic bei einem Fall von Morbus Basedowi mit myasthenie. Mitt Grenzgeb Med Chir 1913;25:746-65.

${ }^{3}$ Blalock A, Mason MF, Morgan HJ, Riven SS. Myasthenia gravis and tumours of the thymic region: report of a case in which the tumour was removed. Ann Surg 1939; 110:544-61.

${ }^{4}$ Keynes G. The surgery of the thymus gland. Br J Surg 1946;33:201-14.

${ }^{5}$ Keynes G. The results of thymectomy in myasthenia gravis. Br Med J 1949;2:611-6.

${ }^{6}$ Keynes G. Surgery of the thymus gland. Second (and third) thoughts. Lancet 1954;i:1197-202.

${ }^{7}$ Keynes G. Investigation into thymic disease and tumour formation. Br J Surg 1955;42:449-62.

${ }^{8}$ Lange MJ. Preparation for and the results of surgery in myasthenia gravis. Br J Surg 1960;48:285-91. 
9 Olanow CW, Lane RJ, Roses AD. Relationship between the acetylcholine receptor antibody titre and the clinical status in myasthenia gravis. Ann NY Acad Sci 1981;377:856-7.

${ }^{10}$ Engel AG, Lambert EH, Santa T. Study of long term anticholinesterase therapy. Effects on neuromuscular transmission and on motor end-plate fine structure. Neurology (Minneap) 1973;23:1273-81.

"Scadding GK, Vincent A, Newsom-Davis J, Henry K. Acetylcholine receptor antibody synthesis by thymic lymphocytes in myasthenia gravis; thymic and peripheral blood cell interactions. Ann NY Acad Sci 1981;377:393-402.

12 Newsom-Davis J, Willcox N, Scadding G, Calder L, Vincent A. Anti-acetylcholine receptor antibody synthesis by cultured lymphocytes in myasthenia gravis; thymic and peripheral blood cell interactions. Ann NY Acad Sci 1981;377:393-402.

${ }^{13}$ Engel WK, Trotter JL, McFarlin DE, McKintosh CL. Thymic epithelial cell contains acetylcholine receptor. Lancet 1977;i:1310-1.

${ }^{14}$ Osserman KE. In: Myasthenia gravis. New York: Grune and Stratton, 1958:80.

is Rowland LP. Controversies about the treatment of myasthenia gravis. J Neurol Neurosurg Psychiatry 1980;43:644-9.
${ }^{16}$ McQuillen MP, Leone M. A treatment carol: thymectomy revisited. Neurology (Minneap) 1977;27: 1103-6.

${ }^{17}$ Perlo VP, Poskanzer DC, Schwab RS, Viets HR, Osserman KE, Genkins G. Myasthenia gravis. Evaluation of treatment in 1,355 patients. Neurology (Minneap) 1966;16:431-39.

${ }^{18}$ Simpson JA. An evaluation of thymectomy in myasthenia gravis. Brain 1958;81:112-44.

${ }^{19}$ Harvey AM. Some preliminary observations on the clinical course of myasthenia gravis before and after thymectomy. Bull NY Acad Med 1948;24:505-22.

${ }^{20}$ Scadding GK, Thomas HC, Havard CWH. Myasthenia Gravis; acetylcholine receptor antibody titres after thymectomy. Br Med J 1977;1:1515.

${ }^{21}$ Emeryk B, Rowinska K, Nowak-Michalska T. Electrophysical investigation (SFEMG) in myasthenia during remission. Communication (Abstract TH8) to 5th Congress on Neuromuscular Diseases, Marseilles, September, 1982.

${ }^{22}$ White MC, De Silva P, Havard CWH. Plasma pyridostigmine levels in myasthenia gravis. Neurology (NY) 1981;31:145-50.

${ }^{23}$ Scadding GK, Sweny P, Wilson SG, Havard CWH, Newsom-Davis J. Glomerulonephritis, thymoma and myasthenia gravis. $Q \mathrm{~J}$ Med 1983;206:187-93. 